\title{
Aloperine activates the Nrf2-ARE pathway when ameliorating early brain injury in a subarachnoid hemorrhage model
}

\author{
SHIBIN SONG, YIMIN CHEN, FENG HAN, MINGHAO DONG, XIN XIANG, \\ JIANMEI SUI, YUMING LI, HUA YANG and JIAN LIU
}

Department of Neurosurgery, Affiliated Hospital of Guizhou Medical University, Guiyang, Guizhou 550004, P.R. China

Received January 29, 2017; Accepted October 13, 2017

DOI: $10.3892 /$ etm.2018.5896

\begin{abstract}
Aloperine (ALO) exhibits neuroprotective effects against oxidative stress in vitro; however, its protective effect in early brain injury (EBI) following experimental subarachnoid hemorrhage (SAH) remains to be elucidated. The aim of the current study was to evaluate the antioxidant activity of ALO in EBI, and its association with nuclear factor erythroid-related factor 2 and the antioxidant responsive element (Nrf2-ARE) survival pathway. In the present study, an experimental SAH model was induced in rats following a prechiasmatic cistern injection. All rats were randomly divided into five groups: Sham, SAH, SAH+ vehicle, and an SAH+ ALO group (including low and high doses). ALO was administrated intraperitoneally at 2 and $24 \mathrm{~h}$ following induction of the $\mathrm{SAH}$ model. Brain samples were collected from each group at $48 \mathrm{~h}$ after SAH induction. Subsequently, western blotting, immunohistochemistry and cell apoptosis assays were performed, along with assessments for brain edema, neurological deficit, and the activity of oxidant/antioxidant factors. It was observed that the expression of Nrf2-ARE pathway-associated agents, including Nrf2, and heme oxygenase-1, were markedly increased in the high concentration ALO group compared with that of the SAH group. In addition, the level of oxidative damage was reduced. Furthermore, early brain damage, including brain edema, neurological deficit and cellular apoptosis were significantly ameliorated. In conclusion, the results of the present study indicate that ALO can ameliorate oxidative damage against EBI following SAH, most likely via the Nrf2-ARE survival pathway.
\end{abstract}

\section{Introduction}

Aneurysmal subarachnoid hemorrhage (aSAH) represents a worldwide health problem which accounts for $5 \%$ of all stroke

Correspondence to: Professor Jian Liu, Department of Neurosurgery, Affiliated Hospital of Guizhou Medical University, 28 Guiyi street, Guiyang, Guizhou 550004, P.R. China

E-mail: liujianmed@126.com

Key words: subarachnoid hemorrhage, early brain injury, nuclear factor erythroid-related factor 2, aloperine cases. Approximately $12 \%$ of the patients affected die before medical care, $33 \%$ within $48 \mathrm{~h}$ and $50 \%$ within 30 days of aSAH. Moreover, $50 \%$ of patients who survive the initial hemorrhage and defeat vasospasm, frequently suffer from permanent disability (1). Traditionally, research has usually focused upon spasm of the large cerebral arteries, which is considered as a determinant element of the complicated pathogenesis of SAH. However, recent studies indicate that the intervention of delayed vasospasm provides little help in the outcome of aSAH patients (2). In these studies, it was suggested that early brain injury (EBI), which is induced in the first $72 \mathrm{~h}$ following initial bleeding, is usually linked to poor outcome, such as brain edema, disruption of the blood-brain barrier (BBB), and a sudden rise in intracranial pressure $(1,3)$.

Accumulating evidence supports the early generation of reactive oxygen species (ROS) and oxidative stress after SAH and their association with EBI and delayed ischemic neurological deficits (DINDs) $(1,3)$. In clinical trials, a reduction in antioxidant systems, and an increase in lipid peroxidation products is found within $72 \mathrm{~h}$ of initial bleeding and correlates well with poor clinical status and outcome (4). Therefore, antioxidants have successfully been used to prevent oxidative stress and reduce EBI in animals $(5,6)$.

Nuclear factor erythroid-related factor 2 (Nrf2), a basic leucine zipper redox-sensitive transcription factor, regulates the expression of many antioxidant and phase II detoxifying enzyme genes, such as heme oxygenase-1 (HO-1), glutathione S-transferase (GST), NADP(H): Quinine oxidoreductase (NQO), UDP-glucuronosyltransferase (UGT), and $\gamma$-glutamylcysteine synthetase $(\gamma \mathrm{GCS})(7)$. Some studies have reported that superoxide can lead to the translocation of $\mathrm{Nrf} 2$ from the cytoplasm to the nucleus, and sequentially binds to the antioxidant responsive element (ARE) following SAH $(8,9)$. Thus, the Nrf2-ARE pathway has been significantly proven to play a neuroprotective role in EBI after SAH, possibly via the inhibition of cerebral oxidative stress by inducing antioxidant and detoxifying enzymes $(8,9)$.

Aloperine (ALO), an alkaloid isolated from Sophora alopecuroides L. (Leguminosae), exerts a variety of pharmacological activities. Previous studies have reported that the protective functions of ALO can be attributed to its anti-inflammatory, anticancer, anti-microbial, anti-viral and anti-allergic effects (10-12). In addition, ALO has been reported to exert significant neuroprotective effects against 
injury caused by oxygen-glucose deprivation and reperfusion (OGD/RP) in vitro, which can be attributed to its antioxidant capacity (12). Significantly, Xu et al (10) had demonstrated that ALO could attenuate neuropathic pain induced by chronic constriction injury via anti-oxidation activity in vivo. Administration of ALO ( 80 and $40 \mathrm{mg} / \mathrm{kg}$, intraperitoneally) obviously increased the SOD concentration and reduced the level of malondialdehyde (MDA) in the spinal cord. However, such antioxidant mechanisms were not investigated in detail in earlier studies. Considering the importance of antioxidant capacity for ALO, the present study aimed to further evaluate the neuroprotective effect of ALO in EBI after SAH and its relationship with the Nrf2-ARE survival pathway.

\section{Materials and methods}

Experimental animals. In total, 150 adult male Sprague-Dawley rats (250-300 g) were used in our study. Animals were purchased from the Animal Department of Guizhou Medical University (Guiyang, China) prior to surgery. Rats were housed under environmentally controlled conditions in a $12 / 12 \mathrm{~h}$ light/dark cycle with a suitable temperature of approximately $25^{\circ} \mathrm{C}$, and provided with free access to food and water. All experimental protocols were approved by the Animal Care and Use Committee of Guizhou Medical University and conformed to the Guide for the Care and Use of Laboratory Animals by the National Institutes of Health.

SAH rat model. Briefly, the rats were anesthetized by an intraperitoneal injection of $10 \%$ chloralic hydrate. The experimental SAH model was induced by the stereotaxic insertion of a needle into the pre-chiasmatic cistern in accordance with a previous study (13). The needle was placed $7.5 \mathrm{~mm}$ anterior to the bregma in the midline at an angle of $45^{\circ}$ in the sagittal plane. Then, $0.3 \mathrm{ml}$ of non-heparinized fresh autologous arterial blood was slowly injected into the pre-chiasmatic cistern for $20 \mathrm{sec}$ with a pump using aseptic technique. After injection, the burr hole was plugged immediately by bone wax, and the rats were maintained in a $30^{\circ}$ head-down position for $30 \mathrm{~min}$. Then, the rats were returned to their cages. Sham animals were subjected to the same procedure exactly as described above with the exception that no blood was injected intra-cisternally.

Experimental design and drug administration. All rats were randomly arranged into five groups as follows: Sham group $(n=30), S A H$ group $(n=30), S A H+$ vehicle group $(n=30)$, $\mathrm{SAH}+$ low concentration ALO group $(\mathrm{n}=30)$, and $\mathrm{SAH}+$ high concentration group ( $\mathrm{n}=30)$. ALO (Kmaels, Shanghai, China), was dissolved in saline containing $10 \%$ acetic acid $(2.5 \mathrm{mg} / \mathrm{ml})(14)$. It was then intra-peritoneally administered to rats at 2 and $24 \mathrm{~h}$ after induction of the SAH model $(75 \mathrm{mg} / \mathrm{kg}$ in the low concentration group and $150 \mathrm{mg} / \mathrm{kg}$ in the high concentration group) (10). Rats in the $\mathrm{SAH}+$ vehicle group were treated with $10 \%$ acetic acid in normal saline as above following SAH insult. Analyses involving western blotting, TUNEL staining, brain edema, immunohistochemistry (IHC), enzyme activity assays and neurological deficits, used five rats from each experimental group.

All animals were killed at a time-point $48 \mathrm{~h}$ after SAH. The rats for Western blot analysis were infused trans-cardially with $250 \mathrm{ml}$ of cold heparinized $0.9 \%$ saline. Then, the inferior basal temporal lobe tissue adjacent to the clotted blood was dissected on ice, and immediately stored in liquid nitrogen immediately until use. The rats for TUNEL staining and IHC were perfused with $100 \mathrm{ml}$ of ice-cold $0.9 \%$ saline through the left ventricle and then fixed with $100 \mathrm{ml} 4 \%$ paraformaldehyde. Then, whole brains were removed and immersed in $4 \%$ paraformaldehyde and stored at $4^{\circ} \mathrm{C}$ until use.

Western blot analysis. Briefly, tissues were mechanically lysed in $20 \mathrm{mM}$ Tris, $\mathrm{pH} 7.6$ containing $0.2 \%$ sodium dodecyl sulfate (SDS), $1 \%$ Triton X-100, $1 \%$ deoxycholate, $1 \mathrm{mM} \mathrm{NaF}$, $2 \mathrm{mM} \mathrm{Na}_{3} \mathrm{VO}_{4}$, and $1 \mathrm{mM}$ phenylmethylsulphonyl fluoride, and centrifuged at $12,000 \mathrm{x} \mathrm{g}$ for $15 \mathrm{~min}$ at $4^{\circ} \mathrm{C}$ for HO- 1 and caspase- 3 analysis. Nuclear protein was extracted for Nrf2 analysis as described previously (15). The concentration of each protein sample was measured by DC protein assay (Beyotime, Nantong, China). Equal amounts of protein per lane was separated by SDS-PAGE (10\% for Nrf2 and HO-1; $12 \%$ for caspase-3), and then transferred to a polyvinylidene-difluoride (PVDF) membrane (Millipore, Darmstadt, Germany). Membranes were then blocked with 5\% skimmed milk for $2 \mathrm{~h}$ at room temperature, and then incubated overnight at $4^{\circ} \mathrm{C}$ with primary antibodies directed against $\mathrm{Nrf} 2(1: 1,000$ dilution; Abcam, Cambridge, MA, USA), caspase-3 (1:1,000 dilution; Cell Signaling Technology, Danvers, MA, USA), HO-1 (1:2,000 dilution; Abcam), and GAPDH (1:5,000 dilution; Bioworld Technology, Inc., St. Louis Park, MN, USA). After washing with Tween-TBS (4x10 min), the membranes were incubated with goat anti-rabbit IgG-horseradish (1:5,000 dilution; Bioworld Technology, Inc.) secondary antibody for $2 \mathrm{~h}$ at room temperature, then washed again $(4 \times 15 \mathrm{~min})$ with Tween-TBS. Finally, bands were visualized by enhanced ECL Western Blot detection reagents (Millipore, Darmstadt, Germany), and exposed to X-ray film (Kodak, Rochester, NY, USA). All experiments were repeated at least three times.

IHC staining. Briefly, formalin-fixed paraffin-embedded sections $(4 \mu \mathrm{m})$ were prepared for IHC staining. Endogenous peroxidase activity was blocked with $3 \% \mathrm{H}_{2} \mathrm{O}_{2}$ for $10 \mathrm{~min}$ followed by blocking serum for $1 \mathrm{~h}$ at room temperature. Sections were then incubated with primary antibodies directed against Nrf2 (1:100 dilution) and HO-1 (1:200 dilution) (both from Abcam) overnight at $4^{\circ} \mathrm{C}$ and then washed with PBS for $15 \mathrm{~min}$. Afterwards, the sections were incubated with horseradish peroxidase (HRP)-conjugated goat anti-rabbit IgG (1:500; SantaS Cruz Biotechnology, Santa Cruz, CA, USA) for $60 \mathrm{~min}$ at room temperature. Diaminobenzidine (DAB) was then used as a chromogen and sections were finally counterstained with haematoxylin. Sections incubated in the absence of primary antibody were used as negative controls.

Two slides (at least $250 \mu \mathrm{m}$ apart) per rat were all randomly selected from the cortex of the temporal lobe from each rat brain. Positive cells were identified with the help of an investigator who was blinded to the experimental groupings.

MDA level and GST-alenzyme activity assay. Frozen samples were homogenized in $10 \mathrm{mM}$ Tris- $\mathrm{HCl}(\mathrm{pH} 7.8)$ and centrifuged for $15 \mathrm{~min}(12,000 \mathrm{x} \mathrm{g})$ and the supernatant collected for assay. MDA, a compound produced during lipid peroxidation, was 
A
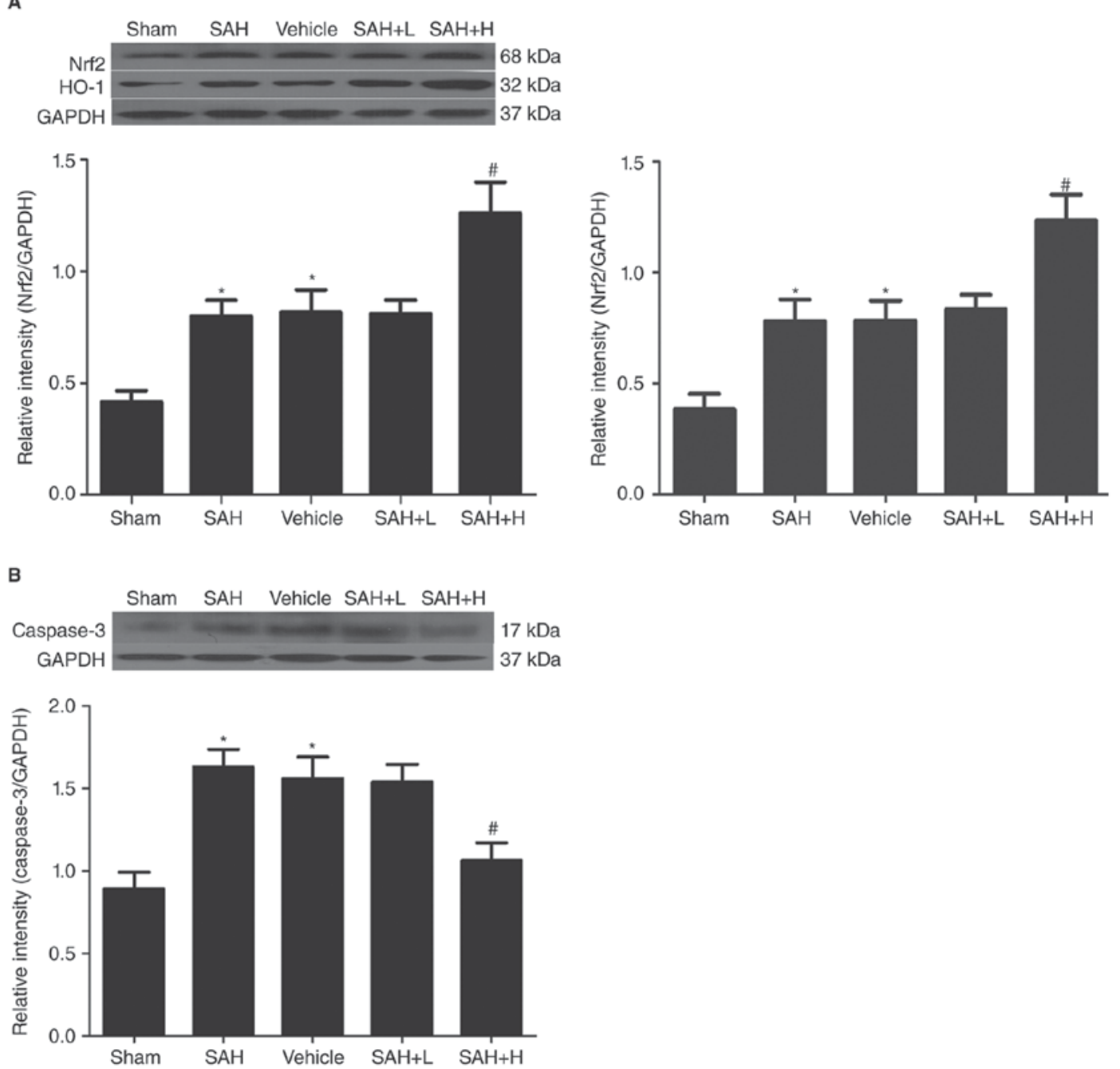

Figure 1. Western blot analysis of Nrf2, HO-1 and caspase-3 levels in the cortex. (A) Different concentrations of ALO influenced the levels of Nrf2, HO-1 at $48 \mathrm{~h}$ after SAH; (B) different concentrations of ALO influenced the levels of caspase- 3 at $48 \mathrm{~h}$ after SAH. SAH+ H, SAH+ high concentration ALO; SAH+ $\mathrm{L}, \mathrm{SAH}+$ low concentration ALO; vehicle, $\mathrm{SAH}+$ vehicle. ${ }^{*} \mathrm{P}<0.05$ vs. sham group, ${ }^{*} \mathrm{P}<0.05$ vs. $\mathrm{SAH}+$ vehicle group ( $\mathrm{n}=5$ per group). Nrf2, nuclear factor erythroid-related factor 2; HO-1, heme oxygenase-1; ALO, aloperine; SAH, subarachnoid hemorrhage.

determined using the thiobarbituric acid method. The given maximum absorbance was $535 \mathrm{~nm}$. GST- $\alpha 1$ enzyme activity was measured via the conjugation reaction of 1-chloro-2,4-dinitrobenzene and glutathione, calculating the slope elevation of extinction coefficient at $340 \mathrm{~nm}$ (9).

TUNEL staining. TUNEL staining was performed following the manufacturer's instructions (Roche Diagnostics, Basel, Switzerland). Three slides (at least $200 \mu \mathrm{m}$ apart) per rat were all randomly selected. Positive cells were identified, counted, and analyzed with the help of an experienced pathologist blinded to the experimental groupings. The extent of brain impairment was evaluated by apoptotic index, which was analyzed by the average number of TUNEL positive cells in each section counted in 10 microscopic fields (at x100 magnification).

Brain water content. Brains were collected at a time-point $48 \mathrm{~h}$ following SAH. The left and right hemisphere brain tissues were immediately weighed (wet weight) and then dried at a temperature of $100^{\circ} \mathrm{C}$ for $48 \mathrm{~h}$ to determine the dry weight. The percentage of water content was calculated with the following formula: [(wet weight - dry weight)/wet weight] $\mathrm{x} 100 \%(16)$.

Neurological deficit. Neurological deficit was performed $1 \mathrm{~h}$ before the rats were euthanized at the $48 \mathrm{~h}$ time-point. Briefly, the neurologic conditions of each rat, such as appetite, activity, and walking deficit, were evaluated according to a scoring scale and the average of the three grades was the neurological deficit. Each rat's appetite was scored as 0, finished meal; 1, left meal unfinished; 2, scarcely ate. Activity assessment was scored as 0 , walk and reach at least three corners of the cage; 1 , walk with some stimulation; 2, almost always lying down. Walking deficit was scored as 0 , no deficits; 1 , unstable walk; 2, unable to walk (17).

Statistical analysis. Data are expressed as mean \pm SEM. All data were analyzed by SPSS 17.0 software (SPSS Inc., Chicago, IL, USA), and statistical differences among groups 

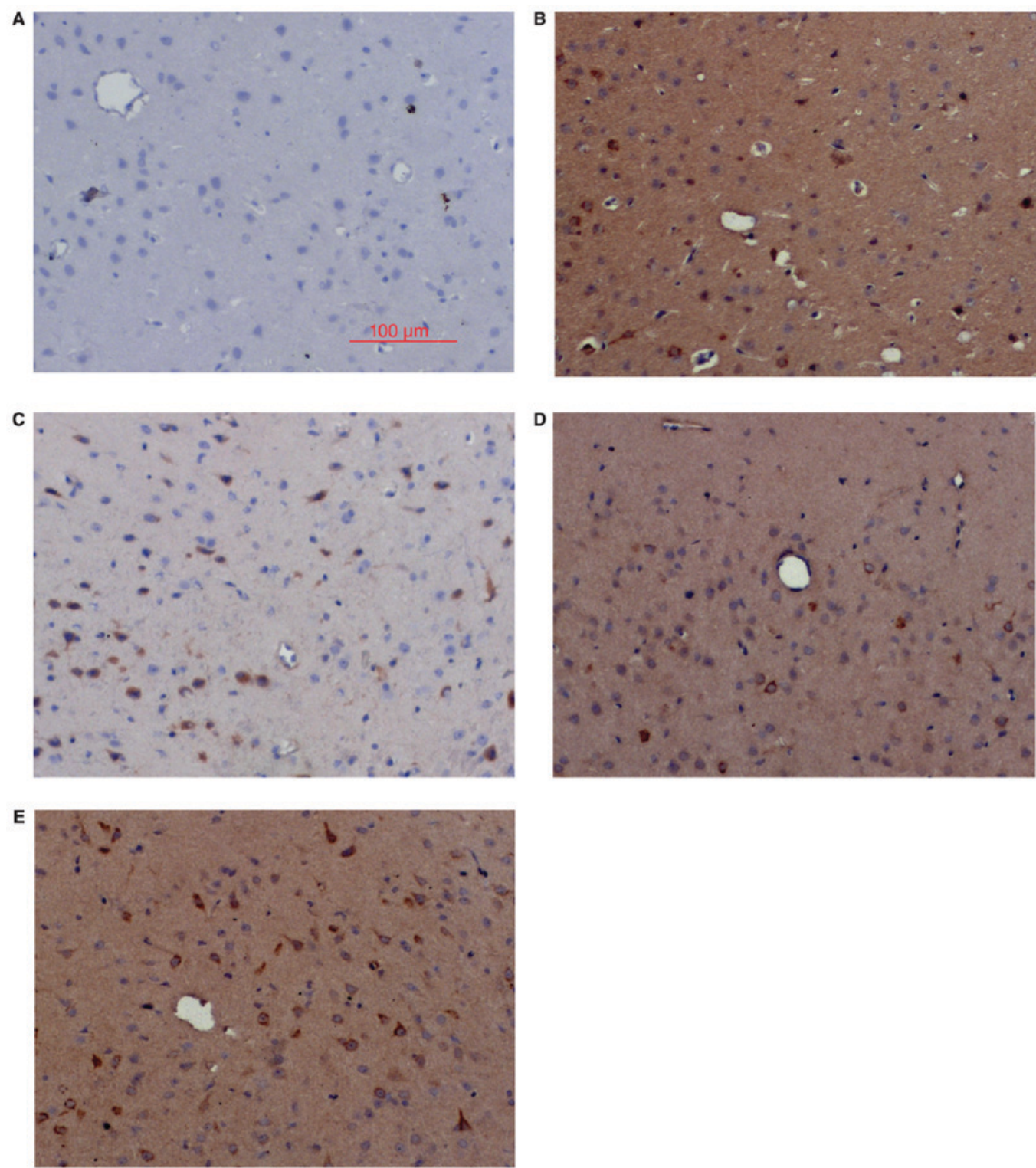

Figure 2. Immunohistochemical analysis of Nrf2 in cortex. (A) sham group; (B) SAH group; (C) SAH+ vehicle group; (D) SAH+ low concentration ALO group; (E) SAH+ high concentration ALO group (scale bar, $100 \mu \mathrm{m}$ ). Nrf2, nuclear factor erythroid-related factor 2; ALO, aloperine; SAH, subarachnoid hemorrhage.

were compared by one-way ANOVA, and differences between groups analysed by Tukey analysis. Statistical significance was accepted when $\mathrm{P}<0.05$.

\section{Results}

Mortality rates of the SAH rat model 48 hafter induction. All the rats in our study survived the procedures of experimental SAH or sham injury. There was no difference among groups in terms of physiological parameters, including body temperature, mean arterial blood pressure, or blood gas analysis (data not shown). Mortality rate was $0(0 / 30$ rats) in the sham group, $16.7 \%(6 / 36$ rats) in the SAH group, $16.7 \%$ (6/36 rats) in the SAH+ vehicle group, $14.3 \%$ (5/35 rats) in the low concentration ALO group, and 11.8\% (4/34 rats) in the high concentration ALO group.

Western blot analysis for $\mathrm{Nrf}$, $\mathrm{HO}-1$ and caspase-3 protein levels. Western blot analysis showed that the protein levels of Nrf2 and HO-1 in the sham group were low and that levels were both significantly increased in the SAH and $\mathrm{SAH}+$ vehicle groups in comparison with the sham group $(\mathrm{P}<0.05$, Fig. 1A). After ALO administration, especially in the high concentration group, the protein levels of Nrf2 and HO-1 were markedly elevated in comparison with the $\mathrm{SAH}+$ vehicle group $(\mathrm{P}<0.05$, Fig. $1 \mathrm{~A})$, but not in the low concentration group $(\mathrm{P}>0.05$, Fig. 1A).

Similarly, compared with the sham group, the expression of caspase- 3 was markedly increased in the SAH and SAH + vehicle groups, but this level was significantly downregulated after high dose ALO treatment ( $\mathrm{P}<0.05$, Fig. 1B).

Nrf2 and HO-1 expression by IHC. Consistent with the western blot results, IHC analysis showed little Nrf2 and HO-1 positive staining in the sham group (Figs. 2 and 3), low levels of positive staining in the $\mathrm{SAH}, \mathrm{SAH}+$ vehicle, and $\mathrm{SAH}+$ low concentration ALO groups (Figs. 2B-D and 3B-D), but 

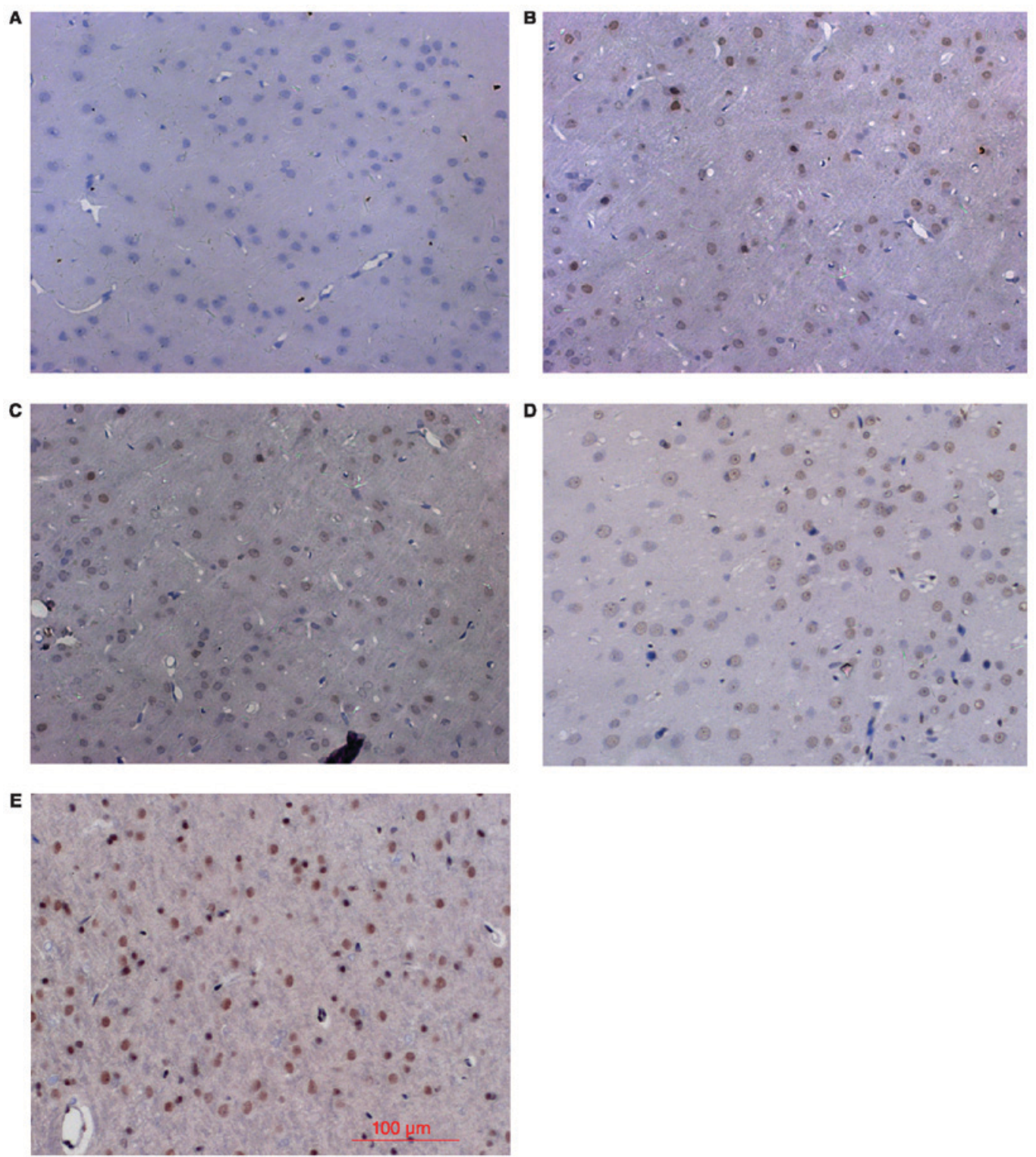

Figure 3. Immunohistochemical analysis of HO-1 in cortex. (A) sham group; (B) SAH group; (C) SAH+ vehicle group; (D) SAH+ low concentration ALO group; (E) SAH+ high concentration ALO group (scale bar, $100 \mu \mathrm{m}$ ). HO-1, heme oxygenase-1; ALO, aloperine; SAH, subarachnoid hemorrhage.

strong positive staining in the SAH+ high concentration ALO group (Figs. 2E and 3E).

MDA levels and GST-alenzyme activity assay. Compared with the sham group, the levels of MDA in the SAH, SAH + vehicle, and $\mathrm{SAH}+$ low concentration ALO groups were obviously increased ( $\mathrm{P}<0.05$, Fig. 4A). However, levels were significantly reduced with high concentration ALO treatment in comparison with the $\mathrm{SAH}+$ vehicle group $(\mathrm{P}<0.05$, Fig. $4 \mathrm{~A})$. Similarly, the enzyme activity of GST- $\alpha 1$ was upregulated following SAH ( $\mathrm{P}<0.05$, Fig. 4B). After ALO administration, especially in the high concentration group, the activity was markedly elevated ( $\mathrm{P}<0.05$, Fig. $4 \mathrm{~B})$, but not in the low concentration group ( $\mathrm{P}>0.05$, Fig. 4B).

Apoptotic death assays. TUNEL-positive apoptotic cells were observed in the temporal lobe with deep brown staining in the nucleus. Few TUNEL positive cells were found in the sham group, and apoptotic index was low (Fig. 5). At $48 \mathrm{~h}$ after initial bleeding, the apoptotic index was obviously increased in the $\mathrm{SAH}$ and $\mathrm{SAH}+$ vehicle groups in comparison with the sham group ( $\mathrm{P}<0.01$, Fig. $5 \mathrm{~B}, \mathrm{C}$ and $\mathrm{F})$. High concentration ALO, but not low concentration ALO treatment, significantly reduced apoptosis in SAH rats, as demonstrated by the low apoptotic index in the SAH + high concentration ALO group $(\mathrm{P}<0.05$, Fig. 5D-F).

Brain edema. Compared with the sham group, obvious increments in brain water content were detected at $48 \mathrm{~h}$ following SAH $(P<0.05$, Fig. 6$)$. The mean value of brain water content was reduced after high concentration ALO administration as compared with the $\mathrm{SAH}+$ vehicle group $(\mathrm{P}<0.05$, Fig. 6).

Neurological deficit. In comparison with the sham group, we found significant impairment of neurological deficit in the $\mathrm{SAH}$ and $\mathrm{SAH}+$ vehicle groups at $48 \mathrm{~h}$ after $\mathrm{SAH}(\mathrm{P}<0.05$; Fig. 7), and there was no difference between the SAH and the SAH+ vehicle groups $(\mathrm{P}>0.05$; Fig. 7). After the high concentration 

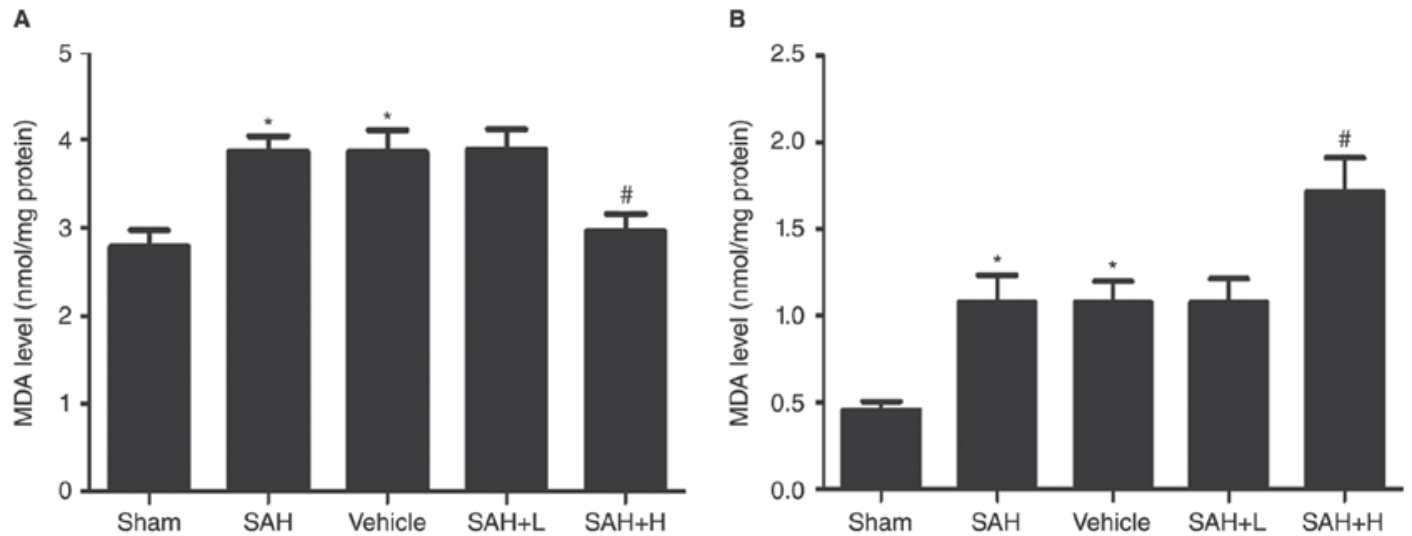

Figure 4. MDA level and GST- $\alpha$ lenzyme activity assays in brain cortex. (A) MDA level; (B) GST- $\alpha$ lenzyme activity. vehicle: SAH+ vehicle; SAH+ H, SAH+ high concentration ALO; $\mathrm{SAH}+\mathrm{L}, \mathrm{SAH}+$ low concentration ALO. ${ }^{*} \mathrm{P}<0.05$ vs. sham group, ${ }^{\text {}} \mathrm{P}<0.05$ vs. SAH+ vehicle group ( $\mathrm{n}=5$ per group). MDA, malondialdehyde; GST, glutathione S-transferase; ALO, aloperine; SAH, subarachnoid hemorrhage.
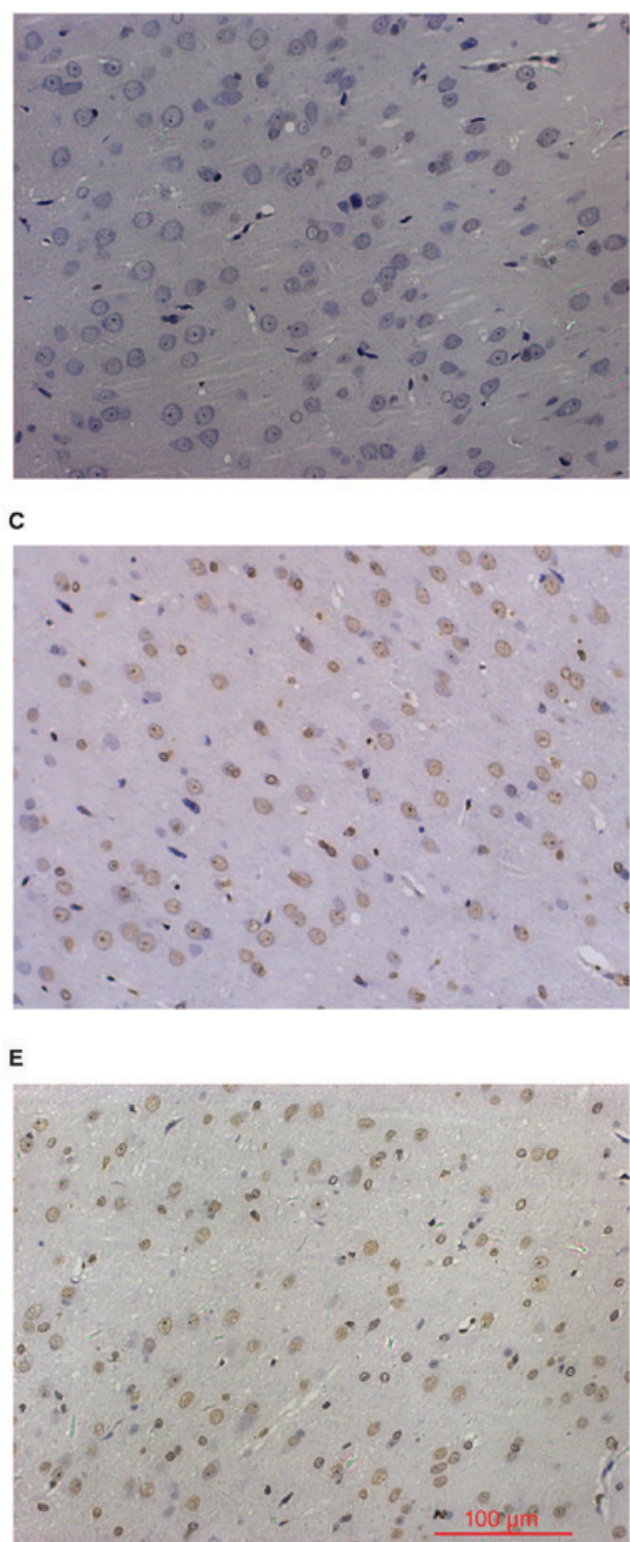

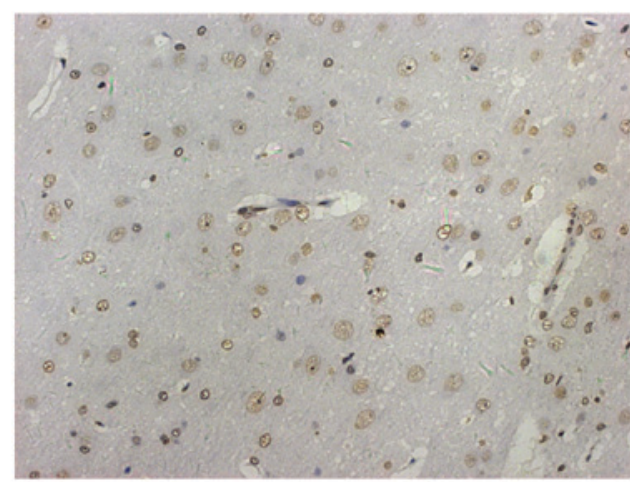

D

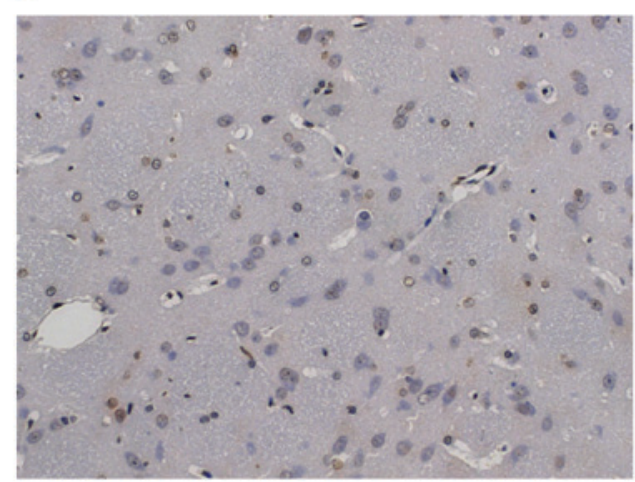

F

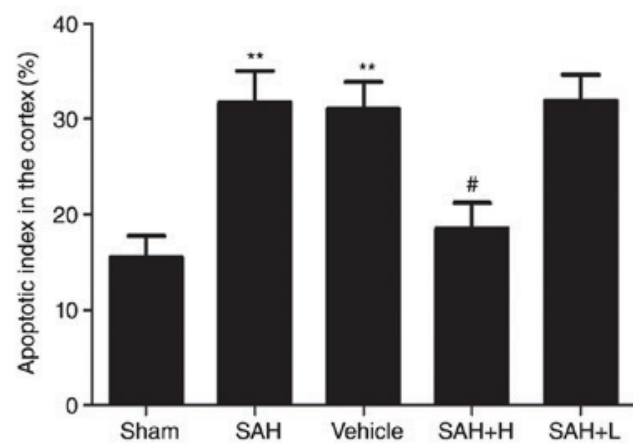

Figure 5. Evaluation of neuronal apoptosis after ALO treatment. (A) Sham group, (B) SAH group, (C) SAH+ vehicle group, (D) SAH+ high concentration ALO group, (E) SAH+ low concentration ALO group. (F) Quantification of apoptosis. Vehicle: SAH+ vehicle; SAH+ H, SAH+ high concentration ALO; $\mathrm{SAH}+\mathrm{L}, \mathrm{SAH}+$ low concentration ALO. ${ }^{*} \mathrm{P}<0.01$ vs. sham group, ${ }^{\sharp} \mathrm{P}<0.05$ vs. SAH+ vehicle group (scale bar, $100 \mu \mathrm{m}, \mathrm{n}=5$ per group). ALO, aloperine; $\mathrm{SAH}$, subarachnoid hemorrhage. 


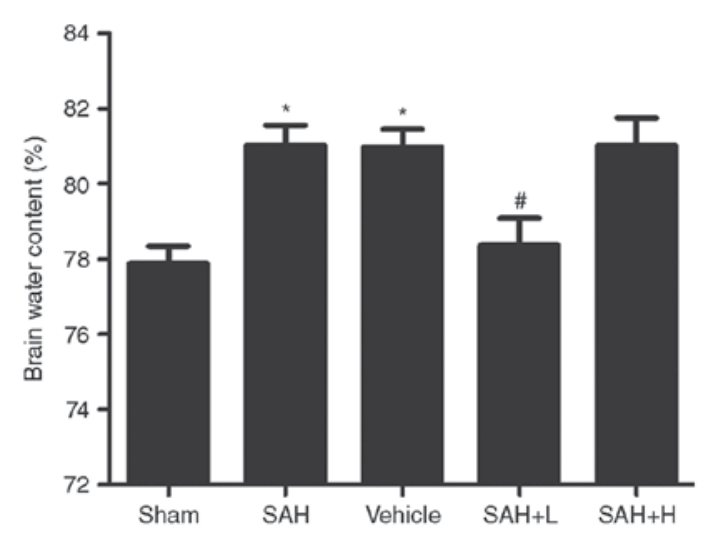

Figure 6. Brain water content at $48 \mathrm{~h}$ after $\mathrm{SAH}$. vehicle, $\mathrm{SAH}+$ vehicle; $\mathrm{SAH}+\mathrm{H}, \mathrm{SAH}+$ high concentration $\mathrm{ALO} ; \mathrm{SAH}+\mathrm{L}, \mathrm{SAH}+$ low concentration ALO. ${ }^{*} \mathrm{P}<0.05$ vs. sham group; ${ }^{\prime} \mathrm{P}<0.05$ vs. $\mathrm{SAH}+$ vehicle group $(\mathrm{n}=5$ per group). ALO, aloperine; SAH, subarachnoid hemorrhage.

ALO treatment, we observed a better level of neurological function. The neurologic score of the high concentration ALO treatment group was significantly lower than that of the $\mathrm{SAH}+$ vehicle group $(\mathrm{P}<0.05$; Fig. 7$)$.

\section{Discussion}

Although it is well established that ALO can confer a neuroprotective effect via attenuating oxidative stress in vitro, our results further suggest that the antioxidative effect of ALO might be attributed to activation of the Nrf2-ARE survival pathway in EBI after SAH.

Factors responsible for the impact of the initial bleeding in SAH include enhanced intracranial pressure (ICP), reduced cerebral blood flow (CBF) and cerebral perfusion pressure (CPP), BBB disruption, brain edema, acute vasospasm and dysfunction of auto-regulation (18). Changes in these factors constitute the EBI period. Despite extensive research, the patient outcome post-aSAH remains poor. The fact that the prevention of delayed cerebral vasospasm (dCVS) does not improve clinical outcome suggests that its importance in patient outcome has been misinterpreted (2). Significantly, EBI has emerged as a new frontier and requires a better understanding and consideration in elaborating therapeutic strategy for improving aSAH outcome $(1,2)$. In our present paper, we created a modified rat model of SAH which has been previously demonstrated to be suitable for pathological and pathophysiological studies of SAH (19). The mortality rate of our SAH model was acceptable, and that the experimental procedure is reproducible and easy to perform. We were able to observe many TUNEL-positive cells (Fig. 5B), an obvious increment in brain edema (Fig. 6), and upregulated caspase-3 levels (Fig. 1B) at $48 \mathrm{~h}$ after initial bleeding, which may partly be ascribed to blood clotting, acute vasospasm, oxidative stress, or acute ischemia injury occurring during the EBI period.

Although the exact mechanism of EBI remains obscure, accumulating evidence has demonstrated that oxidative stress plays an important role in the pathogenesis of EBI. Radicals can cause damage to cardinal cellular components such as lipids, proteins, and nucleic acids, which leads to subsequent cell death by modes of necrosis or apoptosis, resulting in neuronal

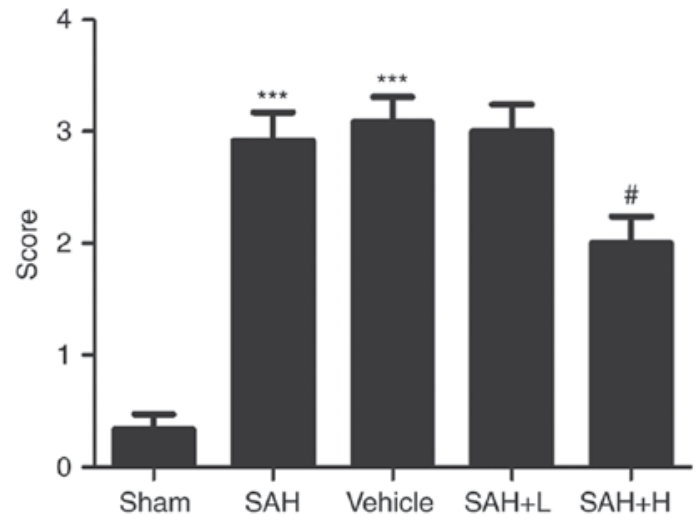

Figure 7. Neurological deficit at $48 \mathrm{~h}$ after $\mathrm{SAH}$. vehicle, SAH+ vehicle; $\mathrm{SAH}+\mathrm{H}, \mathrm{SAH}+$ high concentration ALO; SAH+ L, SAH+ low concentration ALO. ${ }^{* * *} \mathrm{P}<0.001$ vs. sham group; ${ }^{\#} \mathrm{P}<0.05$ vs. $\mathrm{SAH}+$ vehicle group $(\mathrm{n}=5$ per group). ALO, aloperine; SAH, subarachnoid hemorrhage.

damage, cellular apoptosis, endothelial injury, and BBB disruption $(6,20)$. Such damage can become even more widespread because of the weakened cellular antioxidant defense systems. Furthermore, the administration of systemic antioxidants in the experimental SAH model has also been proven to reduce oxidative stress, protect the BBB, alleviate apoptosis, and improve neurological scores $(6,21)$. Therefore, treatment with antioxidants may theoretically act to prevent the propagation of tissue damage and improve both the survival and neurological outcome of SAH. In addition, some important studies have shown that the Nrf2-ARE pathway plays an important role in antioxidant protection in various central nervous system (CNS) diseases, such as cerebral ischemia, traumatic brain injury (TBI), and SAH $(9,15,19,22)$. After initial bleeding, Nrf2 translocates into the nucleus and then binds to ARE, activating a group of cytoprotective enzymes to protect cells against oxidative damage. In the present study, we similarly detected a significant increment of SAH-induced cerebral expression and enzymatic activity of the ARE-mediated downstream factors, HO-1, and GST- $\alpha 1$ (Figs. 1A and 4B). These data were in agreement with findings from a previous study illustrating the activation of Nrf2-ARE pathway at $48 \mathrm{~h}$ following SAH (19).

ALO is a traditional Chinese medicine used for the treatment of cancer. It has been reported to have versatile biological effects, including anti-inflammatory, anti-apoptosis, and anti-oxidation properties $(10,12,23)$. In the current study, we similarly detected that ALO treatment, especially in the high dose group, significantly increased brain Nrf2 and HO-1 levels, and inhibited oxidative stress in SAH rats in comparison with rats in the SAH group (Figs. 1A and 4). Furthermore, statistical analysis between the SAH and the SAH group given a high-concentration of ALO revealed that ALO significantly ameliorated acute injury in EBI, such as brain edema, apoptosis and neurological deficit (Figs. 5-7). These results suggest that the acute administration of ALO could confer an anti-oxidative effect in EBI after SAH, which might be attributed to activation of the Nrf2-ARE survival pathway. Although the anti-oxidative effects of ALO in acute injury have been reported previously $(12,14,23)$, the molecular interaction between these factors is still unknown. Given these diverse actions, it is likely that multiple signaling pathways 
are involved in the protective effect of ALO in acute injury. One vital study suggested that ALO was able to reduce the content of MDA in the hepatic tissue of intoxicated animals but without affecting Superoxide Dismutase (SOD) activity. In another paper, $\mathrm{Hu}$ et al (14) found that ALO could regulate activator protein-1 (AP-1) activity against ischemia reperfusion (IR) -induced renal injury, and thus enhanced SOD expression in order to increase ROS detoxification. These discrepancies might be due to differences in the animal models employed. Interestingly, in CNS studies, it has also been reported that the protective mechanisms of ALO on cultured rat hippocampal neurons injured by $\mathrm{OGD} / \mathrm{RP}$ were related to anti-oxidative stress (12). In this paper, Ma et al (12) found that neurons in the hippocampus from the ALO-group showed significantly reduced MDA and increased endogenous anti-oxidant levels. In the present paper, we detected a similar reduction in MDA level and increment in GST- $\alpha$ lenzyme activity following ALO administration in EBI.

However, the mechanism underlying activation of the Nrf2-ARE pathway by ALO has not been completely elucidated. Some earlier studies have reported that several kinases can activate the Nrf2-ARE pathway in response to some stimuli, including phosphoinositol-3 kinase (PI3K) and extra cellular signal-regulated protein kinase (ERK) $(24,25)$. On the other hand, Hu et al suggested that ALO protects mice against IR-induced renal injury by regulating the PI3K pathway (14). Herein, we speculate that ALO may play a role in the mechanisms underlying activation of the Nrf2-ARE pathway against EBI after SAH insult, which needs further investigation.

Although many compounds with neuroprotective effects against acute injury are in various stages of pharmaceutical development, ALO exhibits unique advantages. Sophora alopecuroides L. and its related species have already experienced thousands of years of human exposure with little reported toxicity and side effects. In addition, ALO can easily diffuse across biological membranes and the BBB in an energy deficient environment (12). Therefore, ALO may be a good candidate drug for the prevention and treatment of SAH-induced acute injury.

It should be noted that there are some limitations to our study. First, we did not explore the effect of ALO upon the Nrf2-ARE pathway in the sham group. Second, the routes of ALO administration usually include intraperitoneal injection, intravenous injection, and oral administration. Here, we only evaluated the effect of intraperitoneal injection. Third, although the improved outcome was regarded as occurring partly by the beneficial effects of ALO by activating the Nrf2-ARE pathway, Nrf2 knockout mice were not used in our study. In conclusion, our results indicate that the acute administration of ALO can ameliorate oxidative stress impairment in EBI, and that this beneficial effect might involve activation of the Nrf2-ARE survival pathway. Our study suggests that ALO may represent a new promising therapeutic agent in the treatment of EBI after SAH.

\section{References}

1. Sehba FA, Hou J, Pluta RM and Zhang JH: The importance of early brain injury after subarachnoid hemorrhage. Prog Neurobiol 97: 14-37, 2012.
2. Hou J and Zhang JH: Does prevention of vasospasm in subarachnoid hemorrhage improve clinical outcome? No. Stroke 44 (6 Suppl 1): S34-S36, 2013.

3. Cahill J, Calvert JW and Zhang JH: Mechanisms of early brain injury after subarachnoid hemorrhage. J Cereb Blood Flow Metab 26: 1341-1353, 2006.

4. Hsieh YP, Lin CL, Shiue AL, Yin H, Morrow JD, Hsu JC, Hsieh TC, Wei HJ and Yen HC: Correlation of F4-neuroprostanes levels in cerebrospinal fluid with outcome of aneurysmal subarachnoid hemorrhage in humans. Free Radic Biol Med 47: 814-824, 2009.

5. Pyne-Geithman GJ, Caudell DN, Prakash P and Clark JF: Glutathione peroxidase and subarachnoid hemorrhage: Implications for the role of oxidative stress in cerebral vasospasm. Neurol Res 31: 195-199, 2009.

6. Gilgun-Sherki Y, Rosenbaum Z, Melamed E and Offen D: Antioxidant therapy in acute central nervous system injury: Current state. Pharmacol Rev 54: 271-284, 2002.

7. Itoh K, Tong KI and Yamamoto M: Molecular mechanism activating Nrf2-Keap1 pathway in regulation of adaptive response to electrophiles. Free Radic Biol Med 36: 1208-1213, 2004.

8. Zhang J, Zhu Y, Zhou D, Wang Z and Chen G: Recombinant human erythropoietin (rhEPO) alleviates early brain injury following subarachnoid hemorrhage in rats: Possible involvement of Nrf2-ARE pathway. Cytokine 52: 252-257, 2010.

9. Wu Q, Zhang XS, Wang HD, Zhang X, Yu Q, Li W, Zhou ML and Wang XL: Astaxanthin activates nuclear factor erythroid-related factor 2 and the antioxidant responsive element (Nrf2-ARE) pathway in the brain after subarachnoid hemorrhage in rats and attenuates early brain injury. Mar Drugs 12: 6125-6141, 2014.

10. Xu YQ, Jin SJ, Liu N, Li YX, Zheng J, Ma L, Du J, Zhou R, Zhao CJ, Niu Y, et al: Aloperine attenuated neuropathic pain induced by chronic constriction injury via anti-oxidation activity and suppression of the nuclear factor kappa B pathway. Biochem Biophys Res Commun 451: 568-573, 2014.

11. Zhao P, Zhou R, Zhu XY, Hao YJ, Li N, Wang J, Niu Y, Sun T, Li YX and Yu JQ: Matrine attenuates focal cerebral ischemic injury by improving antioxidant activity and inhibiting apoptosis in mice. Int J Mol Med 36: 633-644, 2015.

12. Ma NT, Zhou R, Chang RY, Hao YJ, Ma L, Jin SJ, Du J, Zheng J, Zhao CJ, Niu Y, et al: Protective effects of aloperine on neonatal rat primary cultured hippocampal neurons injured by oxygen-glucose deprivation and reperfusion. J Nat Med 69: 575-583, 2015.

13. Ansar S and Edvinsson L: Subtype activation and interaction of protein kinase $\mathrm{C}$ and mitogen-activated protein kinase controlling receptor expression in cerebral arteries and microvessels after subarachnoid hemorrhage. Stroke 39: 185-190, 2008.

14. Hu S, Zhang Y, Zhang M, Guo Y, Yang P, Zhang S, Simsekyilmaz S, Xu JF, Li J, Xiang X, et al: Aloperine protects mice against ischemia reperfusion (IR)-induced renal injury by regulating $\mathrm{PI} 3 \mathrm{~K} / \mathrm{AKT} / \mathrm{mTOR}$ signaling and AP-1 activity. Mol Med: Nov 3, 2015 (Epub ahead of print).

15. Yan W, Wang HD, Feng XM, Ding YS, Jin W and Tang K: The expression of NF-E2-related factor 2 in the rat brain after traumatic brain injury. J Trauma 66: 1431-1435, 2009.

16. Cheng G, Chunlei W, Pei W, Zhen L and Xiangzhen L: Simvastatin activates Akt/glycogen synthase kinase-3beta signal and inhibits caspase-3 activation after experimental subarachnoid hemorrhage. Vascul Pharmacol 52: 77-83, 2010.

17. Yamaguchi M, Zhou C, Nanda A and Zhang JH: Ras protein contributes to cerebral vasospasm in a canine double-hemorrhage model. Stroke 35: 1750-1755, 2004.

18. Ostrowski RP, Colohan AR and Zhang JH: Molecular mechanisms of early brain injury after subarachnoid hemorrhage. Neurol Res 28: 399-414, 2006.

19. Wang Z, Ma C, Meng CJ, Zhu GQ, Sun XB, Huo L, Zhang J, Liu HX, He WC, Shen XM, et al: Melatonin activates the Nrf2-ARE pathway when it protects against early brain injury in a subarachnoid hemorrhage model. J Pineal Res 53: 129-137, 2012.

20. Ayer RE and Zhang JH: Oxidative stress in subarachnoid haemorrhage: Significance in acute brain injury and vasospasm. Acta Neurochir Suppl 104: 33-41, 2008.

21. Ayer RE and Zhang JH: The clinical significance of acute brain injury in subarachnoid hemorrhage and opportunity for intervention. Acta Neurochir Suppl 105: 179-184, 2008. 
22. Ren J, Fan C, Chen N, Huang J and Yang Q: Resveratrol pretreatment attenuates cerebral ischemic injury by upregulating expression of transcription factor $\mathrm{Nrf} 2$ and $\mathrm{HO}-1$ in rats. Neurochem Res 36: 2352-2362, 2011.

23. Wang H, Yang S, Zhou H, Sun M, Du L, Wei M, Luo M, Huang J, Deng H, Feng Y, et al: Aloperine executes antitumor effects against multiple myeloma through dual apoptotic mechanisms. J Hematol Oncol 8: 26, 2015.

24. Zipper LM and Mulcahy RT: Erk activation is required for Nrf2 nuclear localization during pyrrolidine dithiocarbamate induction of glutamate cysteine ligase modulatory gene expression in HepG2 cells. Toxicol Sci 73: 124-134, 2003.
25. Ku BM, Joo Y, Mun J, Roh GS, Kang SS, Cho GJ, Choi WS and Kim HJ: Heme oxygenase protects hippocampal neurons from ethanol-induced neurotoxicity. Neurosci Lett 405: 168-171, 2006.

cc) (i) $\odot$ This work is licensed under a Creative Commons cc) Attribution-NonCommercial-NoDerivatives 4.0 International (CC BY-NC-ND 4.0) License. 